\title{
Simple Model Bonded-Phases to Design a Homogeneous Support for in Silico Chromatography
}

\author{
Toshihiko Hanai* \\ Health Research Foundation, Research Institute for Production Development 4F, 15 \\ Simogamomorimoto-cho, Sakyo-ku, Kyoto, 606-0805.
}

Received:12 December, 2017; Accepted: 24 January, 2018; Published: 03 March, 2018

*Corresponding author: Toshihiko Hanai, Health Research Foundation, Research Institute for Production Development 4F, 15 Simogamomorimotocho, Sakyo-ku, Kyoto, 606-0805; E-mail: thanai@kf7.so-net.ne.jp

\begin{abstract}
The computational chemical calculation clearly demonstrated the difference of reversed-phase, aqueous hydrophilic interaction liquid chromatography and ion-exchange liquid chromatography. The method was applied to study the feasibility of model bonded siloxane and lead phases using capacity ratio $[k]$ of benzoic acid phenol, 4-chlorophenol and benzene measured on octyl-, hexenyl-, hexylamino- and hexylguanidinyl-bonded silica gels in reversed-phase mode liquid chromatography, in acidic eluent. The difference in the molecular interaction strengths were quantitatively analyzed using the calculated energy values. These values indicated the selectivity of these model bonded-phases and the retention mechanisms. These compounds were retained at the alkyl-ligands of these bonded-phases by van der Waals force and the polar groups by hydrogen bonding. The lead [ $\mathrm{SP}^{3}$ orbital element] support that can produce a honey-corn type homogeneous support compare to a polysiloxane support, can be further used in silico chromatography.
\end{abstract}

Keywords: in silico chromatography; quantitative analysis; retention mechanisms; modeling bonded-phases;

\section{Introduction}

Chromatography is a tool to measure molecular interactions, and computational chemistry is a tool to study molecular interactions quantitatively. Now a day, chemically bonded silica gels are inert and stable; therefore, we can exchange data to discuss the retention mechanisms quantitatively. Different chromatographic methods demonstrate the typical molecular interaction $[\mathrm{MI}]$ forces. If we can quantitatively reconstruct the combination of solubility factors, we can quantitatively analyze the chromatographic retention mechanisms [1-3]. The difference is size of molecules, but the molecular interaction forces should be the same. Computational chemical analysis provides the MI energy as the sum of mainly van der Waals [VW], hydrogen bonding [HB], and electrostatic [ES] energy values. The VW energy is related to molecular size; hence, the contact surface area between an analyte and an adsorbent contribute to the MI energy. When HB exists between an analyte and an adsorbent, the HB energy contributes to the MI energy. When ion-ion interactions exist, ES energy contributes to the MI energy. The interaction degree can be obtained as electrostatic energy values as calculated for ion-ion interaction using molecular mechanics $[\mathrm{MM}]$ calculations $[3,4]$.

However, quantitative explanation of chromatographic retention is seemed to be inconclusive due to difficulty of construction of model polysiloxane support with straight alkyl chain blush. Even a model bonded-phase was constructed, but a relation between molecular interactions and chromatographic retention was not demonstrated [5]. It seems that a construction of bonded-phase polysiloxane support was difficult; therefore, the bonded alkyl-chains were independently bent, and did not quantitatively relate with chromatographic retention. Importance of molecular interactions in chromatography was described; however, the quantitative structure retention relationship [QSRR] or quantitative structure property relationship [QSPR] was based on molecular properties, and they did not calculate molecular interactions [6]. Previously alkyl group bonded polysiloxane phases were constructed and the retention times of a variety of compounds, such as phenols, aromatic acids, acidic drugs and basic drugs, have been quantitatively described using the calculated MI energy values using the computational chemical method [in silico] [3, 4]. The basic unit of a polysiloxane support requires six units of silicon dioxide $\left[\mathrm{SiO}_{2}\right]$ to construct a stereo structure of silica gel. However, there is two types of polysiloxane rings exist; therefore, the constructed support produced a garden's ridge like alkyl-blush $[3,7,8]$. Elements with $\mathrm{SP}^{3}$ orbital can produce a honey-corn type homogeneous support, and the supports constructed using carbon and silicon atoms were temporally used to explain the retention mechanism of flat molecules. Because the one side interaction between one side of an analyte and the model bonded phase was related to the molecular interactions. However, such simple model phases were not good enough to describe the retention of drugs whose structure is varied with polar groups. The docking analysis of drugs requires a pocket type phase to increase the contact surface area. Such pocket may imitate the surface of porous silica gels. The docking with a heterogeneous bonded phase based on the polysiloxane support requires training to find the best initial docking location. Therefore, the further search has been achieved 
to construct a honey corn type support. At present, lead [Pb] is the largest $\mathrm{SP}^{3}$ orbital element in my computer system; therefore, a honey corn type support was constructed with bonded alkyl brushes as a model bonded phase silica gels. A remain problem is atomic distance between silanol groups. The minimum distance is $4.638 \AA$ in polysiloxane support. The atomic distance between two $\mathrm{Pb}$ atoms is $4.628 \AA \AA$. It is a little narrow to bond silyl reagents perfectly. Dimethoxy and dimethyl groups of silyl reagents require more space. As the result, large molecules cannot slip into between blushes. Therefore, a bonded phase with a pocket was constructed for further study the molecular interactions.

Previously, the retention time of acidic and neutral compounds was measured using reversed-phase liquid chromatography with an acidic eluent and different bondedsilica gels. The $k$ values measured using the octyl-bonded phases were used as the standard and compared with the $k$ values measured in polar phases [9]. The chromatographic behavior was supported by the MI energy values calculated using the molecular mechanics program [3]. The computational chemical calculation was performed using simple bonded siloxane phases, and the calculated molecular interaction energy values clearly demonstrated the difference of reversed-phase, aqueous hydrophilic interaction liquid chromatography and ion-exchange liquid chromatography [9]. Therefore, the measured $k$ values of benzoic acid, phenol, 4-chlorophenol and benzene on octyl-, hexenyl-, hexylamino- and hexylguanidinyl-bonded silica gels were used to study the feasibility of model bonded $\mathrm{Pb}$ phases.

\section{Experimental}

The model phases were constructed based on model siloxane phases $[3,7]$. Model hexyl [C6]-, octyl [C8]-, hexenyl [C6yl], hexylamino [C6NH2]-, ionized hexylamino [C6NH3]- and hexylguanidyl [C6Gua]-bonded $\mathrm{Pb}$-phases are shown as the examples in Figure 1, where these model phases are indicated as PbC8, PbC6yl, PbC6NH2, PbC6NH3 and PbC6Gua. These bonded $\mathrm{Pb}$-phases are basically the same as the related bondedsiloxane-phases, and these bonded groups are connected via oxygen atom $[9,10]$. The C6- and C8-phases are selected as non-polar phases, and C6yl-phase is selected as a hydrophilic interaction phase [9]. When the C6NH2-phase is ionized based on pKa of alkylamines, the ionized $\mathrm{C6NH2}$-phase is indicated as C6NH3 as an ion-exchanger as C6Gua-phases [ion-exchanger] is. These ion-exchangers also demonstrated for HILIC mode liquid chromatography [9]. The amino- and guanidinyl-bonded phases are used for chromatography of saccharides in HILIC mode [8].

The $\mathrm{Pb}$ atoms were locked [indicate as $\mathrm{L}$ on atoms] as like solid silica gels. These model phases were considered as model phases without silanol effect. Free silanol groups form strong interactions with polar groups. However, modern bonded-phase silica gels synthesized from pure silica gels are chemically well treated and do not practically show the silanol effect. However, popular amino-, cyano-, and phenyl-silica gels are not performed using secondary silanization [end-capping], and thus, a long lifetime is not guaranteed in aqueous solution containing buffer components. When the silanol effect is included, the analysis of chromatographic retention mechanisms becomes very complicated, and the basic mechanisms cannot be quantitatively analyzed. Therefore, silanol effect was not considered in this study, the chromatographic information was based on retention times measured using end-capped bonded-phase silica gels. The analytes were benzene [BZ], phenol [PhOH], 4-chlorophenol [4ClPhOH], and molecular form [BA] and ionized [BAion] benzoic acids. Their capacity ratio, $k$, values were calculated using fructose as the void volume marker [11]. The molecular and ionized forms of benzoic acid were used to make clear the effect of HB and ES energy change. The polar group effect for direct interactions with the model phase was also studied, and either the phenyl group of analytes directly faced toward the model phases [conformation I] or the polar group directly faced toward the model phases [conformation II]. The molecular interaction energy values were calculated using the following equations. The HB, ES, and VW energy values were calculated using the Cache MM program [Fujitsu, Japan]. The computer was a PC model Prime INWIN BL672-4 with Intel Core i7 from Dospara, Yokohama, Japan. These MI energy values $\left[\mathrm{kcal} \mathrm{mol}^{-1}\right]$ are the sum of solute and model phase energy values minus a complex energy value, were calculated per the following equations [8]. MIHB, MIES, and MIVW are MI energy of HB, ES, and VW energy values, respectively.

$\mathrm{MIHB}=\mathrm{HB}[$ molecule A $]+\mathrm{HB}[$ molecule B $]-\mathrm{HB} \quad[$ molecule $A$ and molecule $B$ complex],

MIES $=$ ES [molecule A] + ES [molecule B] - ES [molecule A and molecule B complex],

MIVW = VW [molecule A] + VW [molecule B] - VW

[molecule A and molecule B complex].

The relative MIHB, MIES, and MIVW values indicate the contribution level.

The calculated MI energy values are summarized in Table $1 \& 2$

\section{Results and Discussion}

Benzene retains PbC6, PbC8, PbC6yl and $\mathrm{PbNH} 2$ phases via hydrophobic interaction, where MIVW was about 6 kcal mol-1, and this values are a little higher than those obtained on the model phases using siloxane support, where MIVW were $5-6 \mathrm{kcal} / \mathrm{mol}^{-1}$. The MIVW energy values with ionized NH2 [PbC6NH3] and Guaphase [PbC6Gua] were about $2 \mathrm{kcal} \mathrm{mol}^{-1}$ for the model bonded $\mathrm{Pb}$-phase, and those for the model NH2- and Gua-siloxane were 2 and $5 \mathrm{kcal} \mathrm{mol}^{-1}$, respectively. Such difference may be due to the contact conformation of benzene and guanidyl group. The MIES for the model Gua-bonded siloxane was about $2 \mathrm{kcal} \mathrm{mol}^{-1}$, but the value was about zero for the model PbC6Gua phase.

For phenol, which side of phenol will slips into blush, hydroxyl [conformation II] or phenyl [conformation I] groups? In practical chromatography, the bottom of the blush is considered well endcapped and relatively hydrophobic. Therefore, phenol will slip into the non-polar blush from phenyl-group [conformation I]; however, phenol will slip into the relatively polar blush from the hydroxyl-group [conformation II] or be trapped with the blush polar group. Such probability was also studied by the docking process of phenol, 4-chlorophenol, and benzoic acid. 
Table 1: Relative retention ratios of various compounds on octyl (C8)-, hexenyl (HxNy)-, amino (C6NH2)-, and guanidyl (C6Gua)-phases

\begin{tabular}{|c|c|c|c|c|c|c|c|c|}
\hline \multirow[b]{2}{*}{ Chemicals } & \multirow[b]{2}{*}{$\mathrm{Blog} \mathrm{P}$} & \multirow[b]{2}{*}{$\mathrm{pKa}$} & \multicolumn{3}{|c|}{ pH 3.0} & \multicolumn{3}{|c|}{ pH 9.0} \\
\hline & & & C6yl/C8 & C6NH3/C8 & C6Gua/C8 & C6yl/C8 & C6NH3/C8 & C6Gua/C8 \\
\hline Benzoic acid & 1.61 & 4.20 & 1.37 & 1.32 & 1.46 & 1.21 & 0.42 & 0.04 \\
\hline Phenol & 1.23 & 10.02 & 1.20 & 1.02 & 0.76 & 1.21 & 1.14 & 0.86 \\
\hline 4-Chlorophenol & 1.77 & 9.38 & 1.09 & 0.94 & 0.63 & 1.09 & 1.02 & 0.73 \\
\hline 2,4-Dichlorophenol & 2.25 & 7.89 & 1.06 & 0.89 & 0.55 & 0.98 & 0.68 & 0.61 \\
\hline 2,4,6-Trichlorophenol & 2.80 & 6.42 & 1.05 & 0.90 & 0.47 & 0.89 & 0.51 & 0.52 \\
\hline Benzene & 2.43 & - & 0.95 & 0.89 & 0.45 & 1.01 & 1.03 & 0.54 \\
\hline Toluene & 2.78 & - & 0.91 & 0.83 & 0.37 & 0.97 & 0.94 & 0.45 \\
\hline Ethylbenzene & 3.19 & - & 0.89 & 0.80 & 0.31 & 0.98 & 0.77 & 0.38 \\
\hline
\end{tabular}

Table 2: Molecular interaction energy values $\left(\mathrm{kcal} \mathrm{mol}^{-1}\right)$ calculated using model siloxane- and lead-support phases

\begin{tabular}{|c|c|c|c|c|c|c|c|c|c|c|c|c|c|}
\hline \multicolumn{8}{|c|}{ Siloxane-support } & \multicolumn{6}{|c|}{ Lead-support } \\
\hline Analyte & \multicolumn{4}{|c|}{ Conformation I } & \multicolumn{3}{|c|}{ Conformation II } & \multicolumn{3}{|c|}{ Conformation I } & \multicolumn{3}{|c|}{ Conformation II } \\
\hline $\begin{array}{c}\text { Phase } \\
\text { Benzene }\end{array}$ & $\mathrm{MIHB}^{1}$ & & MIES $^{1}$ & MIVW $^{1}$ & $\mathrm{MIHB}^{2}$ & MIES $^{2}$ & MIVW $^{2}$ & $\mathrm{MIHB}^{1}$ & MIES $^{1}$ & MIVW $^{1}$ & $\mathrm{MIHB}^{2}$ & MIES $^{2}$ & MIVW $^{2}$ \\
\hline C8 & 0.00 & & 0.00 & 5.85 & - & - & - & 0.00 & 0.00 & 5.78 & - & - & - \\
\hline C6yl & 0.00 & & 0.00 & 5.47 & - & - & - & 0.00 & 0.00 & 6.52 & - & - & - \\
\hline C6NH2 & 0.13 & & 0.00 & 5.75 & - & - & - & 0.00 & 0.00 & 5.97 & - & - & - \\
\hline C6NH3 & 9.11 & & 0.00 & 1.96 & - & - & - & 9.11 & 0.01 & 2.11 & - & - & - \\
\hline C6Gua & 12.88 & & 2.01 & 4.77 & - & - & - & 12.01 & 0.06 & 1.94 & - & - & - \\
\hline \multicolumn{14}{|l|}{ Phenol } \\
\hline C6 & 0.01 & & 0.49 & 5.03 & 3.65 & 0.12 & 4.21 & 0.01 & 0.01 & 6.23 & 3.45 & 0.04 & 6.13 \\
\hline $\mathrm{C} 8$ & 0.01 & & 1.41 & 9.42 & 2.55 & 1.52 & 8.64 & 0.00 & 0.01 & 6.11 & 3.44 & 0.03 & 5.98 \\
\hline C6yl & 1.71 & & 0.02 & 5.61 & 3.67 & 0.13 & 4.81 & 1.44 & 0.06 & 6.86 & 3.47 & 0.04 & 7.21 \\
\hline C6NH2 & 0.09 & & 0.13 & 5.98 & 3.83 & 0.05 & 5.77 & 0.02 & 0.02 & 6.27 & 3.44 & 0.04 & 7.21 \\
\hline C6NH3 & 29.08 & & 3.01 & -3.27 & 9.66 & 0.92 & 2.40 & 29.07 & 3.03 & -3.01 & 12.78 & 0.71 & 2.17 \\
\hline C6Gua & 26.00 & & 2.65 & 4.55 & 28.09 & 2.47 & 1.41 & 26.10 & 0.13 & 1.51 & 29.09 & 0.88 & -2.29 \\
\hline \multicolumn{14}{|c|}{ 4-Chlorophenol } \\
\hline C6 & & & 0.05 & 5.96 & 3.64 & 0.16 & 4.79 & 0.00 & -0.02 & 7.21 & 3.38 & 0.06 & 6.49 \\
\hline C8 & & & 1.46 & 10.27 & 3.65 & 1.59 & 9.53 & 0.00 & 0.09 & 6.35 & 3.42 & 0.06 & 6.70 \\
\hline C6NH2 & & & 0.12 & 6.84 & 3.88 & 0.05 & 6.35 & 0.00 & 0.23 & 5.76 & 3.39 & 3.64 & 6.58 \\
\hline C6NH3 & & & -0.22 & 2.46 & 6.30 & 2.73 & 5.47 & 21.43 & 0.37 & 2.72 & 5.58 & 1.59 & 4.59 \\
\hline C6Gua & & & 1.15 & 3.75 & 28.35 & 1.02 & 1.57 & 26.16 & -1.17 & 2.48 & 25.52 & -0.04 & 0.22 \\
\hline
\end{tabular}




\begin{tabular}{|c|c|c|c|c|c|c|c|c|c|c|c|c|}
\hline Benzoic acid & & & & & & & & & & & & \\
\hline C6 & 0.00 & 0.01 & 5.70 & 2.34 & 0.30 & 4.93 & 0.00 & 0.08 & 7.16 & 0.02 & -0.14 & 6.69 \\
\hline C8 & 0.00 & 1.44 & 10.36 & 2.34 & 1.71 & 9.78 & 0.00 & -0.03 & 7.20 & 0.04 & 0.26 & 6.40 \\
\hline C6yl & 1.57 & 0.02 & 6.34 & 2.40 & 0.30 & 5.22 & 0.33 & -0.07 & 7.70 & 2.00 & 0.38 & 6.99 \\
\hline C6NH2 & 3.41 & 0.32 & 5.89 & 2.34 & 0.03 & 4.95 & 3.09 & -0.29 & 5.50 & 2.00 & 0.36 & 6.99 \\
\hline C6NH3 & 23.75 & 12.84 & 2.45 & 9.63 & 0.61 & 3.37 & 23.77 & 13.24 & 0.94 & 9.80 & 1.38 & 3.30 \\
\hline C6Gua & 32.59 & 7.66 & 5.02 & 32.81 & 6.77 & 3.36 & 32.64 & 3.98 & -0.72 & 32.13 & 4.82 & 0.55 \\
\hline
\end{tabular}

\begin{tabular}{|c|c|c|c|c|c|c|c|c|c|c|c|c|}
\hline Ionized benzo & & & & & & & & & & & & \\
\hline C6 & 0.00 & -0.47 & 5.51 & 0.00 & -0.74 & 5.51 & 0.00 & -0.19 & 6.91 & 0.00 & 0.88 & 6.15 \\
\hline C8 & 0.00 & 0.94 & 10.12 & 0.00 & 0.63 & 9.12 & 0.00 & -0.08 & 7.75 & 0.00 & 0.88 & 6.24 \\
\hline C6yl & 0.00 & -0.41 & 5.62 & 0.00 & 0.41 & 6.72 & 0.00 & 0.09 & 7.41 & 0.00 & 1.04 & 7.04 \\
\hline C6NH2 & 0.02 & 1.81 & 6.34 & 0.02 & 1.82 & 5.89 & 0.01 & 2.46 & 6.09 & 0.02 & 1.60 & 6.05 \\
\hline C6NH3 & 29.83 & 116.59 & -8.06 & 29.83 & 116.59 & -8.09 & 32.83 & 119.95 & -8.41 & 32.22 & 119.95 & -8.38 \\
\hline C6Gua & 32.30 & 71.87 & -0.14 & 37.11 & 79.25 & $-3 v .54$ & 35.13 & 75.32 & -3.72 & 39.83 & 82.61 & -6.65 \\
\hline
\end{tabular}

MIHB $^{1}$, MIES $^{1}$,MIVW ${ }^{1}$ : MIHB, MIES, MIVW of conformation I (analyte's phenyl group faces toward support); MIHB $^{2}$,MIES ${ }^{2}$,MIVW ${ }^{2}$ of conformation II (analyte's polar group faces toward support)

On relatively hydrophobic $\mathrm{C6}, \mathrm{C} 8$, and C6yl phases, the phenyl group was mainly interacted with these alkyl-groups of model phases. The MIVW energy values are about 6-7 and 4-9 $\mathrm{kcal} \mathrm{mol}^{-1}$ for $\mathrm{Pb}$ - and siloxane-phases, respectively. The hydroxyl group contacted with the vinyl group of C6nyl with 1.4 and $1.7 \mathrm{kcal} \mathrm{mol}^{-1}$ of MIHB for $\mathrm{Pb}$ - and siloxane phases, respectively. The MIHB energy values are about $3.5 \mathrm{kcal} \mathrm{mol}^{-1}$ while the hydroxyl group moved toward $\mathrm{Pb}$ or siloxane groups [conformation I]. However, the complex of phenol and siloxanephase demonstrated high MIES value $\left[1.45 \mathrm{kcal} \mathrm{mol}^{-1}\right]$ and high MIVW [about $9 \mathrm{kcal} \mathrm{mol}^{-1}$ ]. This behavior was not observed for C10-siloxane phase [the structure is not given], and phenol did not move toward siloxane, and remained at alkyl-group. Therefore, such phenomenon may be due to short ligands that permits the hydroxyl-group can contact with siloxane for less than octyl-group. Practically, the dense bonded-phases are inert by secondary silanization; therefore such conformation may not be occurred, and the main contributed MI energy was MIVW. Therefore, the phenyl group should be contacted with these alkyl ligands. However, the main contributed energy was MIHB for $\mathrm{C} 6 \mathrm{NH} 3$ and C6Gua phases. Even, the phenyl group faced toward these model phases [conformation II], phenol turned the direction, and the hydroxyl group contacted with these ionexchange group. The contribution of MIVW was relatively small. C6NH2 did not show any significant behavior like C6NH3 and C6Gua, rather it demonstrated like an alkyl-bonded phase. If C6NH2 demonstrated like $\mathrm{C} 6 \mathrm{NH} 3$, the eluent should be like ionexchange mode system, but not like normal-phase mode system. The behavior of 4-CIPhOH is like that of $\mathrm{PhOH}$, and the chlorogroup influence was a little in the chromatographic behavior as a hydrophobic compound.

The clear evidence about the contribution of MIES was observed by the comparison of BA and BAion. BA demonstrated strong MIHB with both C6NH3 and C6Gua, and relatively weak contribution of MIES; however, BAion retention is due to ionexchange, thus strong contribution of MIES. The BAion carboxyl group contacted with this anion exchange groups, and neglected hydrophobic interaction of the phenyl group [see Figure $2 \mathrm{~F}$ and
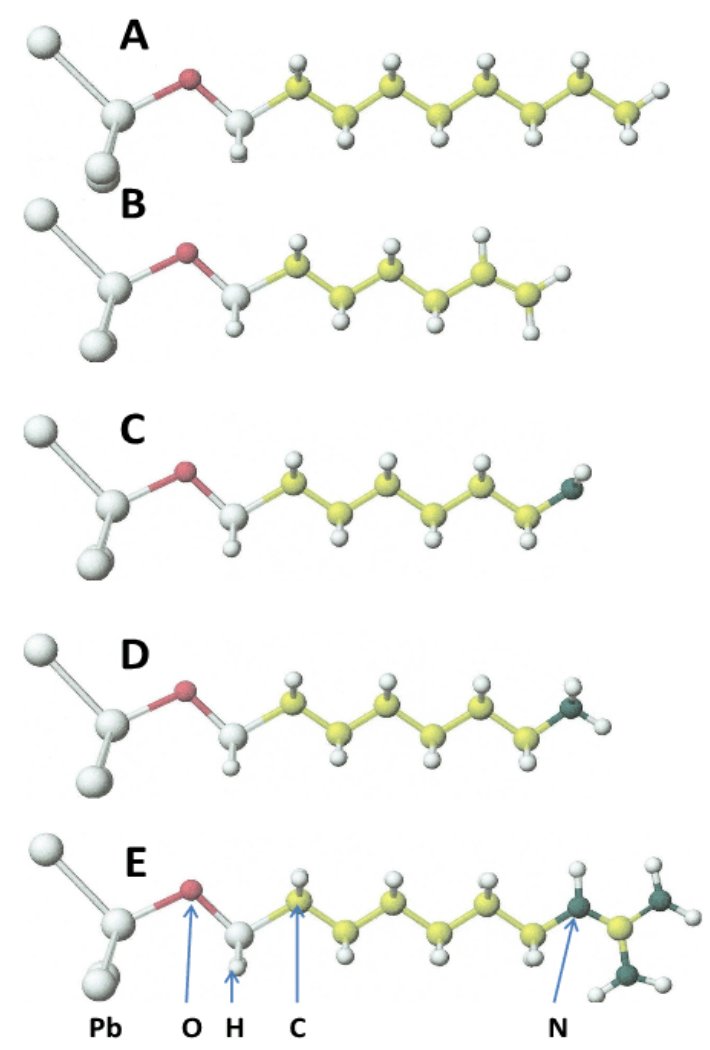

Figure 1: Model Pb-bonded model phases, A: octyl-, B: hexenyl-, C: hexylamino-, D: ionized hexylamino-, and E: hexylguanidyl-bonded lead. 


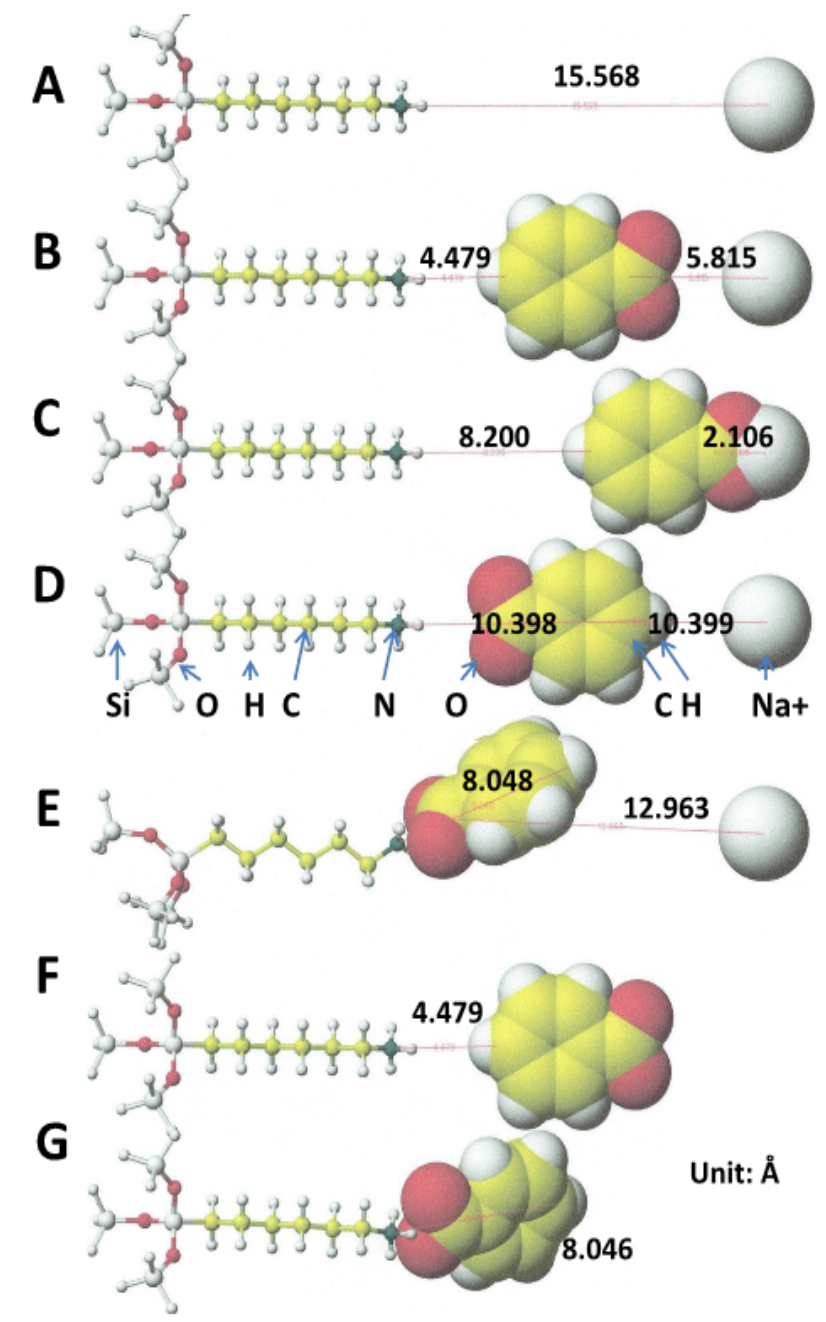

Figure 2: Competitive molecular interactions of ionized benzoic acid with either ionized hexylamino-bonded siloxane or sodium ion, A: original conformation of ionized hexylamino siloxane and sodium ion, B: Before the optimization of the conformation I with ionized benzoic acid, C: optimized conformation B, D: Before the optimization of the conformation II with ionized benzoic acid, E: optimized conformation D, F: Before the optimization of conformation I without sodium ion, G: optimized conformation F; Numbers: atomic distance (unit: $\AA$ ).

Even if the MIES value of C6NH3 and BAion complex was high, retention of BAion at basic eluent was weak. This evidence can be explained by the high MIES value of BAion and $\mathrm{Na}^{+}$complex as shown in Figure 2C. BAion was initially located between C6NH3 and $\mathrm{Na}^{+}$[Figure 2B]. The distance between $\mathrm{N}^{+}$and $\mathrm{Na}^{+}$was $15.6 \AA$ [Figure 2A]. When BAion located as shown as Figure 2D, BAion moved toward C6NH3 [Figure 2E]. However, MIES energy value of Figure 2C [186.9 kcal mol$\left.{ }^{-1}\right]$ was higher than that of Figure 2E [134 $\left.\mathrm{kcal} \mathrm{mol}^{-1}\right]$, and MIHB energy value of Figure 2E was 32.3 kcal mol ${ }^{-1}$ and that of Figure 2C was only $0.01 \mathrm{kcal} \mathrm{mol}^{-1}$. MIHB value of both complexes was negative. This simple demonstration supports the chromatographic behavior of benzoic acid in ionexchange liquid chromatography. The benzoic acid behavior on C6Gua was similar that on C6NH3; however, phenyl of benzoic acid formed face-to-face complex [a parallel complex], and demonstrated high MIHB values over $35 \mathrm{kcal} \mathrm{mol}^{-1}$. This evidence indicates that contribution of MIHB and MIES may be dependent upon properties of ion-exchangers. [Figure 2]

These model phases are not densely bonded phases and are single molecules; therefore, the analyte can form contacts with the siloxane base [9]. The calculated MI energy values cannot be correlated with the measured $\mathrm{k}$ values; however, the calculated MI energy values indicate the contribution of MIHB, MIES, and MIVW. The van der Waals interaction is the main interaction on the C6 and C8 phase. The MIVW values of these analytes were more than $5 \mathrm{kcal} \mathrm{mol}^{-1}$, and the MIHB values were very low [less than $0.02 \mathrm{kcal} \mathrm{mol}^{-1}$ ]. The MIES values were less than $0.2 \mathrm{kcal}$ $\mathrm{mol}^{-1}$. That is ion-exchangers can selectively collect opposition ions and releases the collected ions at presence of strong opposite ions, this is the basic mechanisms of ion-exchange.

On the PbC6yl-phase, the MIVW values of these compounds were $7 \mathrm{kcal} \mathrm{mol}^{-1}$ for both $\mathrm{PhOH}$ and $4 \mathrm{ClPhOH}$. The van der Waals force is the predominant force for the retention of these compounds. In addition, the MIHB values were about $1.7 \mathrm{kcal}$ $\mathrm{mol}^{-1}$ for $\mathrm{BA}, \mathrm{PhOH}$ and $4-\mathrm{ClPhOH}$. When the hydroxyl group slips into the hydrophobic phase [conformation II], MIHB energy value was high [3.4 kcal mol$\left.{ }^{-1}\right]$. However, it should not be occurred except silanol group exists at the site. Carboxyl group of BA contacted with the vinyl group and the MIHB energy value was $2 \mathrm{kcal} \mathrm{mol}^{-1}$. Therefore, the $\mathrm{C} 6 \mathrm{yl} / \mathrm{C} 8$ ratio of BA capacity ratio was 1.3 , and that of $\mathrm{PhOH}$ was 1.1 measured in reversedphase mode was supported by the study using model Pb-phases [8]. The MIHB values supported the retention of compounds with polar compounds retained on the C6yl-bonded phase with hydrophilic interaction liquid chromatography. Practically, the alkyl chain length was 5 methylene unit based on the alkyl-chain contribution to hydrogen bonding of the end polar group [6]. This simple approach may help to study the selectivity of bondedphase silica gels.

\section{Conclusion}

The relative retention times $[k]$ of acidic and neutral compounds were measured using reversed-phase mode liquid chromatography, in acidic eluent, and five different model bondedphase silica gels. Benzoic acid and phenol were retained on the polar phases. The selectivity of the polar phases is supported by the molecular interaction energy values calculated using a molecular mechanics program. These compounds were retained at the alkyl-ligands of these bonded-phases by van der Waals force and the polar groups by hydrogen bonding. The difference in the molecular interaction strengths were quantitatively analyzed using the calculated energy values. These values indicated the selectivity of bonded-phases and the retention mechanisms.

\section{References}

1. Hanai T, Hatano H, Nimura N, Kinoshita T. Computer-aided analysis of molecular recognition in chromatography. Analyst. 1993;118(11): 1371-1374. Doi:10.1039/AN9931801371

2. Hanai T, Hatano H, Nimura N, Kinoshita T. Molecular recognition in chromatography aided by computational chemistry. Supramol Chem. 
1994;3(3): 243-247. Doi: org/10.1080/10610279408028923

3. Hanai T. Quantitative in silico chromatography: Computational modeling of molecular interactions. Cambridge: Royal Society of Chemistry. 2014; 1-338. Doi: org/10.1039/9781782620167

4. Hanai T. Simulation of chromatography of phenolic compounds with a computational chemical method. J Chromatogr A. 2004;1027(1-2): 279-287.

5. Sander L C, Lippa KA, Wise S A. Order and disorder in alkyl stationary phases. Anal Bioanal Chem. 2005;382(3): 646-668. Doi: 10.1007/ s00216-005-3127-2

6. Heinzen V E F, Junkes B d S, Kuhnen C A, Yunes R A. Molecular interactions in chromatographic retention: A tool for QSRR/QSPR/ QSRR studies. In: Aurella Meghea. Molecular interactions. In Tech. 2012; 25-48. Doi: $10.5772 / 37798$
7. Hanai T. Synthesis and properties of stable bonded silica gel packings and the performance. Advances in liquid chromatography. 1996; 307 329. Doi: org/10.1142/9789812831620_0015

8. Hanai T. Introduction of in silico chromatography. J Chromatogr Sep Tech. 2016;7(333): 1-8. Doi:10.4172/2157-7064.1000333

9. Hanai T. Quantitative in silico analysis of HILIC retention mechanisms. Ann Chromatogr Sep Tech. 2017;3(1): 1031-1034

10. Hanai T. Quantitative explanation of retention mechanisms of hydrophobic and hydrophilic-interaction liquid chromatographyinductive effect of alkyl chain. Separation. 2017;4(4): 33. Doi:10.3390/ separations4040033

11. Hanai T. HPLC: A Practical Guide. Cambridge: Royal Society of Chemistry. 1999; 1-134. Doi: org/10.1039/9781847551078 